\title{
RECOVERY OF SLEEP-WAKE CYCLING ON AMPLITUDE-INTEGRATED ELECTROENCEPHALOGRAPHY IN POST-SURGICAL TERM NEONATES
}

\author{
M. Olischar ${ }^{1}$, A. Davidson ${ }^{2}$, R. Hunt ${ }^{3}$ \\ ${ }^{1}$ Neonatology, University Children's Hospital Vienna, Vienna, Austria, ${ }^{2}$ Anaesthesiology, ${ }^{3}$ Neonatology, The \\ Royal Children's Hospital Melbourne, Melbourne, VIC, Australia
}

Background: Sleep characteristics have been used for prediction of neurodevelopmental outcome. Cerebral monitoring has not previously been routinely used to observe effects of anaesthesia on the developing brain.

Aim: To evaluate the influence of anesthesia on recovery of SWC in term newborns after surgery.

Methods: Term neonates admitted to NICU who underwent major intra-abdominal surgery. The Brainz ${ }^{\circledR}$ monitor was applied post-operatively using the standard C3, C4 and P3, P4 positions. Subjects were monitored until mature SWC were observed and the time of onset and quality of SWC together with the background pattern were then assessed. Any neurological abnormality was excluded by neuroimaging.

Results: Fourty-seven patients were recruited (median birthweight 2838 grams). Surgery was performed for congenital diaphragmatic hernia (15), esophageal atresia (12), duodenal and jejunal atresia (7), ileal obstruction (3), exomphalos (3), gastroschisis (4), malrotation (1), meconium ileus (1) and a patent urachus (1). When patients returned to the NICU morphine $(20-60 \mathrm{mcg} / \mathrm{kg} / \mathrm{h})$ and midazolam $(0.5-2.0 \mathrm{mcg} / \mathrm{kg} / \mathrm{min})$ infusions were commenced. Emergence of sleep-wake cycling was observed at a median of 13 hours after surgery (range 1 to 136 hours). SWC became mature at a median of 30 hours (range 4 to 175 hours). All patients were still on morphine and midazolam infusions at this stage.

Conclusion: The emergence of sleep-wake cycling on aEEG after surgery can be observed as early as one hour post-operatively and appears not to be affected by high dose infusions of morphine and midazolam. Relationship between early recovery of sleep-wake cycling and neurodevelopmental outcome remains to be established in follow-up. 